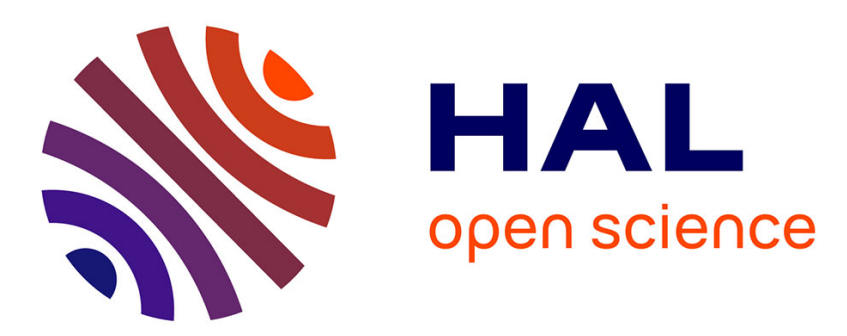

\title{
An Alternative Proof for the Identifiability of Independent Vector Analysis Using Second Order Statistics
}

Dana Lahat, Christian Jutten

\section{- To cite this version:}

Dana Lahat, Christian Jutten. An Alternative Proof for the Identifiability of Independent Vector Analysis Using Second Order Statistics. ICASSP 2016 - 41st IEEE International Conference on Acoustics, Speech and Signal Processing, Mar 2016, Shanghai, China. hal-01212027v2

HAL Id: hal-01212027

https://hal.science/hal-01212027v2

Submitted on 24 Jan 2016

HAL is a multi-disciplinary open access archive for the deposit and dissemination of scientific research documents, whether they are published or not. The documents may come from teaching and research institutions in France or abroad, or from public or private research centers.
L'archive ouverte pluridisciplinaire HAL, est destinée au dépôt et à la diffusion de documents scientifiques de niveau recherche, publiés ou non, émanant des établissements d'enseignement et de recherche français ou étrangers, des laboratoires publics ou privés. 


\title{
AN ALTERNATIVE PROOF FOR THE IDENTIFIABILITY OF INDEPENDENT VECTOR ANALYSIS USING SECOND ORDER STATISTICS
}

\author{
Dana Lahat, Christian Jutten
}

\author{
GIPSA-Lab, UMR CNRS 5216, Grenoble Campus, 38402 Saint-Martin-d'Hères, France
}

\begin{abstract}
In this paper, we present an alternative proof for characterizing the (non-) identifiability conditions of independent vector analysis (IVA). IVA extends blind source separation to several mixtures by taking into account statistical dependencies between mixtures. We focus on IVA in the presence of real Gaussian data with temporally independent and identically distributed samples. This model is always non-identifiable when each mixture is considered separately. However, it can be shown to be generically identifiable within the IVA framework. Our proof differs from previous ones by being based on direct factorization of a closed-form expression for the Fisher information matrix. Our analysis is based on a rigorous linear algebraic formulation, and leads to a new type of factorization of a structured matrix. Therefore, the proposed approach is of potential interest for a broader range of problems.
\end{abstract}

Index Terms - Blind source separation, independent vector analysis, uniqueness, matrix factorization, data fusion

\section{INTRODUCTION}

In this theoretical paper, we present an alternative proof for the identifiability of independent vector analysis (IVA) [1]. By identifiability we mean the minimal set of constraints such that the source estimates are subject only to the unavoidable scale and ordering indeterminacies. Characterizing the uniqueness and identifiability properties of a model is necessary in order to achieve interpretability, i.e., attach physical meaning to its output (e.g., [2, 3]).

The original motivation for IVA was dealing with the arbitrary ordering of the estimated sources in frequency domain blind source separation (BSS) of convolutive mixtures. The dependence across mixtures imposes the estimates of the corresponding sources in all mixtures to be aligned, i.e., have the same permutation [1]. Recently, IVA has shown useful for multimodal data analysis [4] and temporal dynamics [5], among others.

Among the many possible variants of IVA (see, e.g., [6, 7] and references therein), in this paper, we deal only with the identifiability of instantaneous invertible mixtures of real Gaussian random variables with temporally independent and identically distributed (i.i.d.) samples. Our motivation to study this specific setup, proposed by [8], is that each individual mixture is always non-identifiable (e.g., $[9,10])$. Hence, the identifiability results of this specific IVA setting reflect only the added value of the multiset diversity, i.e., the link between datasets $[11,12,13]$. Identifiability in the presence of additional types of diversity is discussed in Section 5. A motivation to focus on second-order statistics (SOS) is that non-identifiability of IVA is associated only with the presence of source samples with

This work is supported by the project CHESS, 2012-ERC-AdG-320684. GIPSA-Lab is a partner of the LabEx PERSYVAL-Lab (ANR-11-LABX0025).
Gaussian distribution [6, 14]. Furthermore, the relative simplicity and mathematical tractability of this model allow us to reformulate our derivations in form of a new type of factorization of a structured matrix, which may be useful beyond this specific task.

In this paper, $\cdot{ }^{\top}, \cdot{ }^{-\top},{ }^{H}$ denote transpose, inverse transpose, and Hermitian, respectively. Scalars, vectors and matrices are denoted as normal $(\lambda, M)$, bold lower $(\boldsymbol{\lambda}, \mathbf{x})$ or upper case $(\mathcal{H}, \mathbf{A})$ symbols, respectively. The $(k, l)$ th entry of $\mathbf{P}$ is denoted $p^{[k, l]}$ and the $k$ th entry of $\boldsymbol{\lambda}$ is $\lambda^{[k]} . \circledast, \otimes, \odot$, denote the Hadamard, Kronecker and column-wise Khatri-Rao products, respectively. $\operatorname{diag}\{\boldsymbol{\lambda}\}$ is a matrix whose diagonal is $\boldsymbol{\lambda}$, whereas $\operatorname{diag}\{\mathbf{X}\}$ is a diagonal matrix by setting to zero the off-diagonal terms of a square matrix $\mathbf{X} . \mathbf{I}_{M}$ and $\mathbf{0}_{M \times N}$ denote an identity or all-zero matrix, respectively.

The rest of this paper is organized as follows. The IVA model and its corresponding notion of identifiability are defined in Section 2. In Section 3, we present the principal concepts behind the previous proofs, and then explain the underlying idea of ours. Section 4 is dedicated to the actual mathematical analysis, and presents our proof in detail. It is concluded with a short discussion of the meaning of these results. Section 5 concludes our work with a broader view of uniqueness and identifiability, especially in the context of data fusion.

\section{IVA: PROBLEM STATEMENT}

\subsection{Model}

Consider $T$ observations of $K$ vectors $\mathbf{x}^{[k]}(t)$, modelled as

$$
\mathbf{x}^{[k]}(t)=\mathbf{A}^{[k]} \mathbf{s}^{[k]}(t) \quad 1 \leq t \leq T, 1 \leq k \leq K,
$$

where $\mathbf{A}^{[k]}$ are $M \times M$ invertible matrices that may be different $\forall k$, and $\mathbf{x}^{[k]}(t)$ and $\mathbf{s}^{[k]}(t)$ are $M \times 1$ vectors. For a fixed $k$, each mixture (1) corresponds to classical BSS. In a basic IVA model, the elements of the $K \times 1$ vector $\mathbf{s}_{i}(t)=\left[s_{i}^{[1]}(t), \ldots, s_{i}^{[K]}(t)\right]^{\top}, i=$ $1, \ldots, M$, are statistically dependent whereas the pairs $\left(\mathbf{s}_{i}(t), \mathbf{s}_{j}(t)\right)$ are statistically independent for all $i \neq j \in\{1, \ldots, M\}$. Therefore, IVA aims at extracting $M$ mutually independent vector elements (whence its name) $\mathbf{s}_{i}(t), i=1, \ldots, M$, from $K$ sets of measurements $\mathbf{x}^{[k]}(t), k=1, \ldots, K$, by exploiting not only the statistical independence within each set of measurements but also the dependence between sets of measurements. In fact, some of these assumptions can be relaxed while maintaining the identifiability of the model $[6,14,15,16]$. In this respect, the goal of this paper is to re-prove some of these relaxations.

In this paper, we focus on separation by exploiting the statistical (in)dependencies that are manifested in the SOS. The covariance between the $i$ th and $j$ th sources in the $k$ th and $l$ th data sets, respectively, can be collected either in the $(i, j)$ th element of the covariance matrix $\mathbf{S}^{[k, l]} \triangleq E\left\{\mathbf{s}^{[k]}(t) \mathbf{s}^{[l] H}(t)\right\} \in \mathbb{C}^{M \times M}, k, l=1, \ldots, K$, 
which is diagonal due to the statistical independence for each $i \neq j$, or in the $(k, l)$ th element of $\mathbf{S}_{i i} \triangleq E\left\{\mathbf{s}_{i}(t) \mathbf{s}_{i}^{H}(t)\right\} \in \mathbb{C}^{K \times K}$. In what follows, we assume that $\mathbf{S}_{i i}$ are invertible $\forall i$. The goal of SOSbased IVA can thus be stated as estimating the sources, given the set of covariance matrices of the observations $\mathbf{X}^{[k, l]}=\mathbf{A}^{[k]} \mathbf{S}^{[k, l]} \mathbf{A}^{[l] \top}$. As already shown by $[6,14,15]$, and will be re-explained in this paper, identifiability of this specific IVA model consists only in characterizing a minimal set of additional constraints on the source covariance matrices.

\subsection{Uniqueness and Identifiability}

Consider now two alternative representations to (1). In the first one, all observations are collected into one vector $\mathbf{x}(t)=$ $\left[\mathbf{x}^{[1] \top}(t), \ldots, \mathbf{x}^{[K]^{\top}}(t)\right]^{\top}$. It can readily be verified that $\mathbf{x}(t)=$ $\sum_{i=1}^{M}\left(\mathbf{I}_{K} \odot \mathbf{A}_{i}\right) \mathbf{s}_{i}(t)$, where $\mathbf{A}_{i} \triangleq\left[\mathbf{a}_{i}^{[1]}|\cdots| \mathbf{a}_{i}^{[K]}\right]$ is an $M \times K$ matrix, and $\mathbf{a}_{i}^{[k]}$ is the $i$ th column of $\mathbf{A}^{[k]}$. It follows that the ordering of the sources is the same in all data sets. In the second representation, we rewrite each mixture as $\mathbf{x}^{[k]}(t)=\sum_{i=1}^{M} \mathbf{a}_{i}^{[k]} s_{i}^{[k]}(t)$. It is clear that the pairs $\left(\mathbf{a}_{i}^{[k]}, s_{i}^{[k]}(t)\right)$ and $\left(\alpha^{-1} \mathbf{a}_{i}^{[k]}, \alpha s_{i}^{[k]}(t)\right)$ are indistinguishable $\forall \alpha \neq 0$. If the model is subject only to these trivial scale and ordering indeterminacies, we say that it is unique. Since all $\mathbf{A}^{[k]}$ are invertible, uniqueness implies that the entire model is identifiable. The goal of this paper is to characterize the minimal conditions on this model such that it is unique and identifiable.

\section{BACKGROUND: PREVIOUS PROOFS VS. OUR APPROACH}

A first proof was presented by Vía et al. [15], who study SOSbased IVA in the complex domain. Their proof is based directly on the definition of identifiability, by characterizing the admissible set of uniquely-defined solutions of the IVA model, up to the unavoidable indeterminacies. The derivation is based on the observation that for normalized sources, i.e., $\mathbf{S}^{[k, k]}=\mathbf{I}_{M}$, the trivial indeterminacies boil down to $\mathbf{S}^{[k, l]}=\mathbf{Q}^{[k]} \widetilde{\mathbf{S}}^{[k, l]} \mathbf{Q}^{[l]}$, where $\mathbf{Q}^{[k]} \in \mathbb{C}^{M \times M}$ are unitary matrices, and both $\mathbf{S}^{[k, l]}$ and $\widetilde{\mathbf{S}}^{[k, l]}$ are elements of the admissible set. By reformulating this relationship as $\boldsymbol{\Sigma}^{[k, l]}=\mathbf{Q}^{[k]} \widetilde{\boldsymbol{\Sigma}}^{[k, l]} \mathbf{Q}^{[k]}$, where $\boldsymbol{\Sigma}^{[k, l]} \triangleq \mathbf{S}^{[k, l]}\left(\mathbf{S}^{[k, l]}\right)^{H}$ and $\widetilde{\mathbf{\Sigma}}^{[k, l]} \triangleq \widetilde{\mathbf{S}}^{[k, l]}\left(\widetilde{\mathbf{S}}^{[k, l]}\right)^{H}$, the authors show that each column of $\mathbf{Q}^{[k]}$ contains exactly one non-null entry. Eventually, the theorem that they provide corresponds to Scenario 1 (Section 4.2), generalized to the complex case, but only with $D=K$. They mention that additional identifiability conditions can be found in the case of vanishing correlation values.

A second proof was proposed by Anderson et al. [6, 14]. Their identifiability analysis is quite comprehensive, as it admits nonGaussian, non-stationary and statistically dependent samples, all in the real domain. Their approach is to determine the necessary and sufficient conditions under which the Fisher information matrix (FIM) is positive definite. Their proof is based on a sequence of statements, starting from the claim that non-positive-definiteness of the FIM for each pair of sources $(i, j)$, formulated as

$$
\mathbf{F}_{i, j}=\operatorname{Cov}\left\{\left[\begin{array}{c}
\operatorname{diag}\left\{\boldsymbol{\Psi}_{i} \mathbf{Z}_{j}^{\top}\right\} \\
\operatorname{diag}\left\{\boldsymbol{\Psi}_{j} \mathbf{Z}_{i}^{\top}\right\}
\end{array}\right]\right\} \in \mathbb{R}^{2 K \times 2 K}
$$

amounts to non-positive-definiteness of its sample estimate, that is, if and only if (iff) $\exists(\boldsymbol{\mu}, \boldsymbol{\nu}) \neq(\mathbf{0}, \mathbf{0})$ such that

$$
\operatorname{diag}\left\{\boldsymbol{\Psi}_{i} \mathbf{Z}_{j}^{\top}\right\} \boldsymbol{\mu}-\operatorname{diag}\left\{\boldsymbol{\Psi}_{j} \mathbf{Z}_{i}^{\top}\right\} \boldsymbol{\nu}=0
$$

where $\mathbf{Z}_{i}$ and $\widehat{\mathbf{Z}}_{i}$ are $K \times T$ matrices whose $(k, t)$ th element is the source sample $s_{i}^{[k]}(t)$ or its estimate, respectively, and $\boldsymbol{\Psi}_{i} \triangleq$ $\boldsymbol{\Psi}_{i}\left(\widehat{\mathbf{Z}}_{i}\right)=\frac{\partial \log \left(p_{i}\left(\widehat{\mathbf{Z}}_{i}\right)\right)}{\partial \widehat{\mathbf{Z}}_{i}} \in \mathbb{R}^{K \times T}$ is the multivariate score function. This analysis eventually leads to a general expression for IVA identifiability [6, Theorem 1], from which, as explained in [6, 14], various special cases can be derived, including all those in Section 4.2, which correspond to our model.

In this paper, we propose a different approach, which is based on finding the necessary and sufficient conditions for non-positivedefiniteness of the FIM by direct factorization of the closed-form expression of the pairwise FIM. In [17], it is shown that the inverse of the $2 K \times 2 K$ symmetric matrix

$$
\mathcal{H}=\left[\begin{array}{cc}
\mathbf{S}_{j j} \circledast \mathbf{S}_{i i}^{-1} & \mathbf{I}_{K} \\
\mathbf{I}_{K} & \mathbf{S}_{j j}^{-1} \circledast \mathbf{S}_{i i}
\end{array}\right]
$$

is the key ingredient in the Cramér-Rao lower bound (CRLB) on the estimation error of the oblique projection matrices, which extract the sources from their mixtures. Hence, (4) corresponds to the (pairwise) FIM. Therefore, the identifiability of the model consists in characterizing the sufficient and necessary conditions for the invertibility of $\mathcal{H}$. Although this idea may be reminiscent of the approach of $[6,14]$, our analytical derivation is in fact more similar to that in [18, Appendix B]: we factorize the explicit closed-form FIM directly, using algebraic identities.

We mention that an expression similar to (4), but with sample estimates of the covariance matrices, appears in [19, Equation (9)]. However, neither [19] nor [15] mention any explicit link of this matrix to the performance of the model or its identifiability. Expression (4) is a special case of the more general result in [17], in which the mutually independent elements within each mixture are multivariate.

\section{IDENTIFIABILITY: ALTERNATIVE PROOF}

\subsection{Analyzing $\mathcal{H}$}

For $\mathcal{H}$ to be positive-definite, we require that for any non-zero vector $\mathbf{x} \in \mathbb{R}^{2 K \times 1}, \mathbf{x}^{\top} \mathcal{H} \mathbf{x}>0$. Conversely, for $\mathcal{H}$ to be non-positivedefinite, there must exist some non-zero $\mathbf{x} \in \mathbb{R}^{2 K \times 1}$ such that

$$
0=\mathbf{x}^{\top} \mathcal{H} \mathbf{x}=\mathbf{x}^{\top} \mathbf{V}^{\top} \mathbf{V} \mathbf{x} \quad \Leftrightarrow \quad \mathbf{V} \mathbf{x}=\mathbf{0}
$$

Based on (5), we look for a meaningful decomposition $\mathcal{H}=\mathbf{V}^{\top} \mathbf{V}$. The desired factorization

$$
\mathcal{H}=\underbrace{\left[\begin{array}{ll}
\left(\mathbf{S}_{j j}^{\frac{1}{2} \top} \odot \mathbf{S}_{i i}^{-\frac{1}{2}}\right)^{\top} \\
\left(\mathbf{S}_{j j}^{-\frac{1}{2}} \odot \mathbf{S}_{i i}^{\frac{1}{2} \top}\right)^{\top}
\end{array}\right]}_{\mathbf{V}^{\top}} \underbrace{\left[\begin{array}{lll}
\mathbf{S}_{j j}^{\frac{1}{2} \top} \odot \mathbf{S}_{i i}^{-\frac{1}{2}} & \mathbf{S}_{j j}^{-\frac{1}{2}} \odot \mathbf{S}_{i i}^{\frac{1}{2} \top}
\end{array}\right]}_{\mathbf{V} \in \mathbb{R}^{K \times 2 K}}
$$

follows from introducing the square root factorization $\mathbf{S}_{j j}=$ $\mathbf{S}_{j j}^{\frac{1}{2}} \mathbf{S}_{j j}^{\frac{1}{2} \top}$, and then applying Identity 1 (Appendix 6.1).

Next, we find $\mathbf{x} \neq \mathbf{0}$ such that $\mathbf{V x}=\mathbf{0}$. Without loss of generality, we look for $\mathbf{x}$ in the general form $\mathbf{x} \triangleq\left[\begin{array}{ll}\boldsymbol{\mu}^{\top} & -\boldsymbol{\nu}^{\top}\end{array}\right]^{\top}$ where $\boldsymbol{\mu}$ and $\boldsymbol{\nu}$ are $K \times 1$ vectors. $\mathbf{V x}=\mathbf{0}$ implies that

$$
\left(\mathbf{S}_{j j}^{\frac{1}{2} \top} \odot \mathbf{S}_{i i}^{-\frac{1}{2}}\right) \boldsymbol{\mu}-\left(\mathbf{S}_{j j}^{-\frac{1}{2}} \odot \mathbf{S}_{i i}^{\frac{1}{2} \top}\right) \boldsymbol{\nu}=\mathbf{0}
$$

for some non-zero $\boldsymbol{\mu}$ and/or $\boldsymbol{\nu}$ [note the difference between (6) and (3)]. We now turn to finding these $\boldsymbol{\mu}$ and $\boldsymbol{\nu}$. Using Identity 2 (Appendix 6.1), equality (6) rewrites as

$$
\operatorname{vec}\left\{\mathbf{S}_{i i}^{-\frac{1}{2}} \operatorname{diag}\{\boldsymbol{\mu}\} \mathbf{S}_{j j}^{\frac{1}{2}}\right\}=\operatorname{vec}\left\{\mathbf{S}_{i i}^{\frac{1}{2} \top} \operatorname{diag}\{\boldsymbol{\nu}\} \mathbf{S}_{j j}^{-\frac{1}{2} \top}\right\} .
$$


Removing the "vec" notation and using the invertibility of $\mathbf{S}_{i i}^{\frac{1}{2}}$ and $\mathbf{S}_{j j}^{\frac{1}{2}}$, we obtain

$$
\operatorname{diag}\{\boldsymbol{\mu}\} \mathbf{S}_{j j}=\mathbf{S}_{i i} \operatorname{diag}\{\boldsymbol{\nu}\} .
$$

Hence, non-identifiability exists for non-zero $(\boldsymbol{\mu}, \boldsymbol{\nu})$ for which equality (7) holds.

The identifiability problem (7) can be further simplified into characterizing all the solutions to

$$
\operatorname{diag}\{\boldsymbol{\lambda}\} \mathbf{R}=\mathbf{P} \operatorname{diag}\{\boldsymbol{\lambda}\}
$$

where $\boldsymbol{\lambda}$ is an arbitrary $K \times 1$ vector (instead of the original problem with a $2 K \times 1$ vector $\mathbf{x}$ ). The $K \times K$ symmetric positive definite matrices $\mathbf{P}$ and $\mathbf{R}$ are normalized versions of $\mathbf{S}_{i i}$ and $\mathbf{S}_{j j}$ such that their diagonals are the identity, $p^{[k, k]}=1=r^{[k, k]} \forall k$. The normalization scheme is

$$
\mathbf{P}=\boldsymbol{\Omega}_{i i} \mathbf{S}_{i i} \boldsymbol{\Omega}_{i i}^{\top} \quad \text { and } \quad \mathbf{R}=\boldsymbol{\Omega}_{j j} \mathbf{S}_{j j} \boldsymbol{\Omega}_{j j}^{\top}
$$

where $\boldsymbol{\Omega}_{i i} \triangleq \operatorname{diag}\left\{\left(\mathbf{S}_{i i}\right)^{-\frac{1}{2}}\right\} \forall i$. The proof of (8) is given in Section 6.2 .

In order to extract further information from (8), we multiply (8) either on the left or on the right by $\operatorname{diag}\{\boldsymbol{\lambda}\}$,

$$
\begin{aligned}
\operatorname{diag}\{\boldsymbol{\lambda}\} \mathbf{R} \operatorname{diag}\{\boldsymbol{\lambda}\} & =\mathbf{P} \operatorname{diag}^{2}\{\boldsymbol{\lambda}\} \\
\operatorname{diag}\{\boldsymbol{\lambda}\} \mathbf{P} \operatorname{diag}\{\boldsymbol{\lambda}\} & =\operatorname{diag}^{2}\{\boldsymbol{\lambda}\} \mathbf{R}
\end{aligned}
$$

Since the LHS of (10) is symmetric, so must be the RHS:

$$
\begin{aligned}
\operatorname{diag}^{2}\{\boldsymbol{\lambda}\} \mathbf{P} & =\mathbf{P} \operatorname{diag}^{2}\{\boldsymbol{\lambda}\} \\
\operatorname{diag}^{2}\{\boldsymbol{\lambda}\} \mathbf{R} & =\mathbf{R} \operatorname{diag}^{2}\{\boldsymbol{\lambda}\}
\end{aligned}
$$

which can be reformulated as the set of equations $\forall k, l$

$$
\begin{aligned}
& \left(\lambda^{2[k]}-\lambda^{2[l]}\right) p^{[k, l]}=0 \\
& \left(\lambda^{2[k]}-\lambda^{2[l]}\right) r^{[k, l]}=0
\end{aligned}
$$

where $\lambda^{2[k]} \triangleq\left(\lambda^{[k]}\right)^{2}$. Note that neither $k=l$ nor $\lambda^{2[k]}=\lambda^{2[l]}$ yields any constraints.

\subsection{Necessary and Sufficient Conditions for Identifiability}

It remains to characterize the non-identifiability scenarios associated with $\boldsymbol{\lambda} \neq \mathbf{0}$. Non-zero $\boldsymbol{\lambda}$ is associated with two different types of non-identifiability, as we now explain (a similar type of analysis is used, e.g., in [20, Chapter 4.1]).

Scenario 1. The first type is associated with

$$
2 \leq D \leq K: \underbrace{\lambda^{[1]}=\ldots=\lambda^{[D]}}_{\neq 0} \neq \underbrace{\lambda^{[k>D]}}_{\text {whatever }}
$$

i.e., the first $D \geq 2$ elements of $\boldsymbol{\lambda}$ are equal, non-zero and different from the rest. Such $\boldsymbol{\lambda}$ are associated with non-identifiability where there exists a pair $(i, j)$ of sources whose covariance matrices have the structure

$$
\begin{array}{r}
\mathbf{S}_{i i}=\left[\begin{array}{c|c}
\mathbf{S}_{i i}^{[1: D, 1: D]} & \mathbf{0}_{D \times(K-D)} \\
\hline \mathbf{0}_{(K-D) \times D} & \mathbf{S}_{i i}^{[D+1: K, D+1: K]}
\end{array}\right] \\
\mathbf{S}_{j j}=\left[\begin{array}{c|c}
\mathbf{S}_{j j}^{[1: D, 1: D]} & \mathbf{0}_{D \times(K-D)} \\
\hline \mathbf{0}_{(K-D) \times D} & \mathbf{S}_{j j}^{[D+1: K, D+1: K]}
\end{array}\right]
\end{array}
$$

and the blocks on the top left are related to each other by

$$
\mathbf{S}_{i i}^{[1: D, 1: D]}=\operatorname{diag}\{\boldsymbol{\omega}\} \mathbf{S}_{j j}^{[1: D, 1: D]} \operatorname{diag}\{\boldsymbol{\omega}\}
$$

where $\boldsymbol{\omega}$ is a $D \times 1$ vector with strictly positive values, and $\mathbf{S}_{i i}^{[1: D, 1: D]}$ is the $D \times D$ sub-matrix of $\mathbf{S}_{i i}$ with row and column indices running from 1 to $D$ (similar to Matlab notation). The proof of Scenario 1 is given in Section 6.3.

Scenario 2. The second type is associated with

$$
1 \leq D \leq K: \underbrace{\lambda^{[1]} \neq \ldots \neq \lambda^{[D]}}_{\text {one may be zero }} \neq \underbrace{\lambda^{[k>D]}}_{\text {whatever }}
$$

i.e., the first $D \geq 1$ elements of $\boldsymbol{\lambda}$ are different from each other and also from the rest. Such $\boldsymbol{\lambda}$ are associated with non-identifiability where there exists a pair $(i, j)$ of sources whose covariance matrices have the structure

$$
\begin{array}{r}
\mathbf{S}_{i i}=\left[\begin{array}{c|c}
\operatorname{diag}\left\{\mathbf{S}_{i i}^{[1: D, 1: D]}\right\} & \mathbf{0}_{D \times(K-D)} \\
\hline \mathbf{0}_{(K-D) \times D} & \mathbf{S}_{i i}^{[D+1: K, D+1: K]}
\end{array}\right] \\
\mathbf{S}_{j j}=\left[\begin{array}{c|c}
\operatorname{diag}\left\{\mathbf{S}_{j j}^{[1: D, 1: D]}\right\} & \mathbf{0}_{D \times(K-D)} \\
\hline \mathbf{0}_{(K-D) \times D} & \mathbf{S}_{j j}^{[D+1: K, D+1: K]}
\end{array}\right]
\end{array}
$$

The proof of (16) is given in Section 6.4.

Rigorous examination of all other types of $\boldsymbol{\lambda}$ that are not characterized by (13) or (15) shows that they are either (i) subsumed under scenarios 1-2 by reordering the source or mixture indices, or (ii) do not lead to any other non-identifiable scenarios.

One can readily verify that there is one-to-one correspondence between the identifiability conditions in Scenarios 1-2 and those in $[6,14,15]$, for our model. We have thus completed our (alternative) proof.

\subsection{Discussion}

We conclude this section with a brief discussion of these identifiability results. Most of these interpretations have already been mentioned in $[6,14,15]$, and are revisited here in view of our reformulation, as well as for the sake of completeness.

In terms of source separation, Scenario 1 implies that the IVA model is non-identifiable if in $D \geq 2$ mixtures there exist two sources $(i, j)$ which (i) are not correlated with any of the other $(K-D)$ mixtures, as imply the zeros on the off-block-diagonal, and (ii) (14c) holds. Scenario 1 deals with non-identifiability when there is a specific type of similarity between the covariance and crosscovariance profiles of two sources, across mixtures. Therefore, it can be regarded as the multiset analogue of the "spectral diversity" constraint for BSS, when non-stationarity or colour diversity is concerned; see, e.g., [11]. For $D=K$, Scenario 1 corresponds to [15, Theorem 1] and [6, Theorem 3]. For $D \geq 2$, this is the case of factorizable probability density function (pdf) in [6, Section VII]. For $K=2$, SOS IVA amounts to generalized eigenvalue decomposition (GEVD) [21]. One can readily verify that the GEVD is indeed not unique when Scenario 1 holds.

Scenario 2 implies that the IVA model is non-identifiable if in each of the mixtures indexed $k=1, \ldots, D$, there exist two sources $(i, j)$ that do not have any statistical correlation with any of the other $(K-1)$ mixtures, as imply the zeros on the off-diagonal. The non-identifiability of this scenario finds its counterpart in the well-known notion that in classical BSS, one cannot separate more 
than one Gaussian source (e.g., [22]). Indeed, for $K=1$, this nonidentifiable scenario amounts to a single BSS problem with (at least two) Gaussian sources [6, Theorem 5]. If the structure (16) holds for all pairs of sources $i, j=1, \ldots, N$, then this scenario amounts to the presence of $D$ individual BSS mixtures; scenario 2 validates the known fact that this setup is not identifiable using SOS alone.

In terms of data fusion, Scenario 1 implies that if a pair of sources is linked across at least two data sets, then these sources are generically identifiable. Both scenarios indicate that nonidentifiability is associated with anomalous, or non-generic, behaviour that is shared by a pair of sources, across one or more data sets. This pairwise characteristic is not so surprising, since nonidentifiability essentially means that two (or more) sources cannot be distinguished. A possible remedy to Scenario 1 is adding data sets, which may reduce the risk of (14c) to occur. A possible remedy to both non-identifiability scenarios is to exploit additional types of diversity, for example non-stationarity or non-flat spectra, at least in the mixtures that contain problematic pairs of sources.

\section{CONCLUSION}

In this paper, we presented an alternative proof for the identifiability of a special case of IVA. The proposed approach is based on a rigorous linear algebraic formulation. This type of analysis, as well as the new matrix factorization associated with it, may turn out useful in a broader range of problems. As a first step in this direction, we have recently applied this same analytical approach to a generalization of IVA to multidimensional components; the results in this paper become a special case of those in [16].

In this work, we discussed only SOS-based IVA in which each individual BSS problem was not identifiable. We mention that analogous results, which show that an ensemble of certain types of tensor decompositions can have a unique decomposition even if each tensor cannot be uniquely decomposed individually, can be found in [23]. As noted, e.g., by [6, 14, 15] (with some concrete mathematical proofs therein), additional types of diversity such as higherorder statistics (HOS), complex values, and non-stationarity, may further improve IVA identifiability. Therefore, rigorous analysis of uniqueness and identifiability of various coupled models, with different types and combinations of diversity, is an ongoing challenge that is not limited to IVA. For data fusion, there is particular interest in such results, as a large number of questions regarding the combination of various modalities and data sets is still largely open; see, e.g., $[13$, Section V.E] and references therein.

\section{APPENDIX}

\subsection{Some Algebraic Properties}

Identity 1. Consider four matrices $\mathbf{A} \in \mathbb{R}^{\mu \times \xi}, \mathbf{B} \in \mathbb{R}^{\nu \times \xi}, \mathbf{C} \in$ $\mathbb{R}^{\mu \times \eta}, \mathbf{D} \in \mathbb{R}^{\nu \times \eta}$. Then,

$$
\mathbf{A}^{\top} \mathbf{C} \circledast \mathbf{B}^{\top} \mathbf{D}=(\mathbf{A} \odot \mathbf{B})^{\top}(\mathbf{C} \odot \mathbf{D}) .
$$

Proof. Directly, by evaluating the $(i, j)$ th element at each side.

The special case $\mathbf{A}=\mathbf{C}, \mathbf{B}=\mathbf{D}$ can be found, e.g., in [24, Chapter 3.2, Equation (12)].

Identity 2. For any matrices $\mathbf{A}, \mathbf{B}$ and vector $\mathbf{x}$ with appropriate dimensions,

$$
(\mathbf{B} \odot \mathbf{A}) \mathbf{x}=\operatorname{vec}\left\{\mathbf{A} \operatorname{diag}\{\mathbf{x}\} \mathbf{B}^{\top}\right\}
$$

\subsection{Proof of $\operatorname{diag}\{\boldsymbol{\lambda}\} \mathbf{R}=\mathbf{P} \operatorname{diag}\{\boldsymbol{\lambda}\}$}

Applying the normalization scheme (9) to (7) and changing sides,

$$
\underbrace{\boldsymbol{\Omega}_{i i} \operatorname{diag}\{\boldsymbol{\mu}\} \boldsymbol{\Omega}_{j j}^{-1}}_{\triangleq \operatorname{diag}\{\boldsymbol{\lambda}\}} \mathbf{R}=\mathbf{P} \underbrace{\boldsymbol{\Omega}_{i i}^{-\top} \operatorname{diag}\{\boldsymbol{\nu}\} \boldsymbol{\Omega}_{j j}^{\top}}_{\triangleq \operatorname{diag}\{\boldsymbol{\varphi}\}}
$$

Since $\mathbf{P}$ and $\mathbf{R}$ are normalized, the $k$ th diagonal term of (19) is $\lambda^{[k]}=\varphi^{[k]} \forall k$. Hence, $\operatorname{diag}\{\boldsymbol{\lambda}\}=\operatorname{diag}\{\boldsymbol{\varphi}\}$. Applying this insight to (19) concludes our proof.

\subsection{Proof of Scenario 1}

We begin by the special case $D=K$ which is the simplest. Let $\boldsymbol{\lambda}=\lambda \mathbf{1}_{K}, \lambda \neq 0$ such that $\operatorname{diag}\{\boldsymbol{\lambda}\}=\lambda \mathbf{I}_{K}$. Then, (8) reduces to $\lambda \mathbf{I R}=\mathbf{P I} \lambda$, which implies $\mathbf{R}=\mathbf{P}$. Next, it follows from (9) that

$$
\boldsymbol{\Omega}_{j j}^{-1} \boldsymbol{\Omega}_{i i} \mathbf{S}_{i i} \boldsymbol{\Omega}_{i i} \boldsymbol{\Omega}_{j j}^{-1}=\mathbf{S}_{j j}
$$

The latter can be reformulated as

$$
\operatorname{diag}\{\boldsymbol{\omega}\} \mathbf{S}_{i i} \operatorname{diag}\{\boldsymbol{\omega}\}=\mathbf{S}_{j j}
$$

where $\operatorname{diag}\{\boldsymbol{\omega}\} \triangleq \boldsymbol{\Omega}_{j j}^{-1} \boldsymbol{\Omega}_{i i}$ such that $\boldsymbol{\omega}$ is a $K \times 1$ vector with arbitrary strictly positive values. We now prove the general case. When (13) holds, (12) implies that

$$
\begin{aligned}
& p^{[k \leq D, l \leq D]}(\underbrace{\lambda^{2[k \leq D]}-\lambda^{2[l \leq D]}}_{0})=0 \\
& p^{[k \leq D, l>D]}(\underbrace{\lambda^{2[k \leq D]}-\lambda^{2[l>D]}}_{\neq 0})=0 \\
& p^{[k>D, l \leq D]}(\underbrace{\lambda^{2[k>D]}-\lambda^{2[l \leq D]}}_{\neq 0})=0 \\
& p^{[k>D, l>D]}(\underbrace{\lambda^{2[k>D]}-\lambda^{2[l>D]}}_{\text {depends on } \boldsymbol{\lambda}})=0
\end{aligned}
$$

which induces a structure

$$
\mathbf{P}=\left[\begin{array}{c|c}
\mathbf{P}^{[1: D, 1: D]} & \mathbf{0}_{D \times(K-D)} \\
\hline \mathbf{0}_{(K-D) \times D} & \mathbf{P}^{[D+1: K, D+1: K]}
\end{array}\right]
$$

and similarly for $\mathbf{R}$. That is, a set of equal values within $\boldsymbol{\lambda}$ induces a diagonal sub-block of entries in $\mathbf{P}$ and $\mathbf{R}$, which are uncorrelated with the rest. In a second step, within this $D \times D$ block, denoting $\lambda^{[k \leq D]}=\lambda$, (8) implies that $\lambda \mathbf{I}_{D} \mathbf{R}^{[1: D, 1: D]}=\lambda \mathbf{I}_{D} \mathbf{P}^{[1: D, 1: D]}$, which implies $\mathbf{R}^{[1: D, 1: D]}=\mathbf{P}^{[1: D, 1: D]}$. Undoing the normalization of (23) via (9), similarly to (20), we obtain the desired result.

\subsection{Proof of Scenario 2}

When (15) holds, using a procedure similar to (22), equations (12) induce a structure

$$
\mathbf{P}=\left[\begin{array}{c|c}
\mathbf{I}_{D} & \mathbf{0}_{D \times(K-D)} \\
\hline \mathbf{0}_{(K-D) \times D} & \mathbf{P}^{[D+1: K, D+1: K]}
\end{array}\right]
$$

i.e., the top left block is the identity, the top right and bottom left blocks are zero. Only the bottom right block is unaffected; and similarly for R. Undoing the normalization of (24) via (9) yields (16), which concludes our proof.

Identity 2 can be found, e.g., in [25, Table III, T3.13]. 


\section{REFERENCES}

[1] T. Kim, T. Eltoft, and T.-W. Lee, "Independent vector analysis: An extension of ICA to multivariate components," in Independent Component Analysis and Blind Signal Separation. 2006, vol. 3889 of $L N C S$, pp. 165-172, Springer Berlin Heidelberg.

[2] R. B. Cattell, “"'Parallel proportional profiles” and other principles for determining the choice of factors by rotation," Psychometrika, vol. 9, no. 4, pp. 267-283, Dec. 1944.

[3] R. A. Harshman, "Foundations of the PARAFAC procedure: models and conditions for an "explanatory" multimodal factor analysis," UCLA Working Papers in Phonetics, vol. 16, pp. 1-84, Dec. 1970.

[4] Y. Levin-Schwartz, V. D. Calhoun, and T. Adal1, "Data-driven fusion of EEG, functional and structural MRI: A comparison of two models," in Proc. CISS, Princeton, NJ, USA, Mar. 2014, pp. 1-6.

[5] S. Ma, V. D. Calhoun, R. Phlypo, and T. Adalı, "Dynamic changes of spatial functional network connectivity in healthy individuals and schizophrenia patients using independent vector analysis," NeuroImage, vol. 90, pp. 196-206, Apr. 2014.

[6] M. Anderson, G.-S. Fu, R. Phlypo, and T. Adalı, "Independent vector analysis: Identification conditions and performance bounds," IEEE Trans. Signal Process., vol. 62, no. 17, pp. 4399-4410, Sep. 2014.

[7] M. Congedo, R. Phlypo, and J. Chatel-Goldman, "Orthogonal and non-orthogonal joint blind source separation in the leastsquares sense," in Proc. EUSIPCO, Bucharest, Romania, Aug. 2012, pp. 1885-1889.

[8] Y.-O. Li, T. Adal1, W. Wang, and V. D. Calhoun, "Joint blind source separation by multiset canonical correlation analysis," IEEE Trans. Signal Process., vol. 57, no. 10, pp. 3918-3929, Oct. 2009.

[9] J. J. Lacoume and P. Ruiz, "Sources indentification: a solution based on the cumulants," in 4th Annual ASSP Workshop on Spectrum Estimation and Modeling, Minneapolis, MN, USA, Aug 1988, pp. 199-203.

[10] J.-F. Cardoso, "Blind signal separation: Statistical principles," Proc. IEEE, vol. 86, no. 10, pp. 2009-2025, Oct. 1998.

[11] J.-F. Cardoso, "The three easy routes to independent component analysis; contrasts and geometry," in Proc. ICA, San Diego, CA, USA, Dec. 2001, pp. 1-6.

[12] T. Adal1, M. Anderson, and G.-S. Fu, "Diversity in independent component and vector analyses: Identifiability, algorithms, and applications in medical imaging," IEEE Signal Process. Mag., pp. 18-33, May 2014.
[13] D. Lahat, T. Adal1, and Ch. Jutten, "Multimodal data fusion: An overview of methods, challenges and prospects," Proc. IEEE, vol. 103, no. 9, pp. 1449-1477, Sep. 2015.

[14] M. Anderson, Independent Vector Analysis: Theory, Algorithms, and Applications, Ph.D. thesis, Graduate School of the University of Maryland, Baltimore County, 2013.

[15] J. Vía, M. Anderson, X.-L. Li, and T. Adalı, "Joint blind source separation from second-order statistics: Necessary and sufficient identifiability conditions," in Proc. ICASSP, Prague, Czech Republic, May 2011, pp. 2520-2523.

[16] D. Lahat and Ch. Jutten, "A generalization to Schur's lemma with an application to joint independent subspace analysis," Tech. Rep. hal-01247899, GIPSA-Lab, Grenoble, France, Dec. 2015.

[17] D. Lahat and Ch. Jutten, "Joint independent subspace analysis using second-order statistics," IEEE Trans. Signal Process., in press.

[18] D. Lahat, J.-F. Cardoso, and H. Messer, "Identifiability of second-order multidimensional ICA," in Proc. EUSIPCO, Bucharest, Romania, Aug. 2012, pp. 1875-1879.

[19] J. Vía, M. Anderson, X.-L. Li, and T. Adalı, "A maximum likelihood approach for independent vector analysis of Gaussian data sets," in Proc. MLSP, Beijing, China, Sep. 2011.

[20] D. Vvedensky, "Group theory course notes," http://www.cmth.ph.ic.ac.uk/people/d. vvedensky/courses.html, 2001.

[21] T. W. Anderson, An introduction to multivariate statistical analysis, John Wiley \& Sons, 1958.

[22] P. Ruiz and J.-L. Lacoume, "Extraction of independent sources from correlated inputs a solution based on cumulants," in Workshop on Higher-Order Spectral Analysis, Vail, CO, USA, 28-30 Jun. 1989, pp. 146-151.

[23] M. Sørensen and L. De Lathauwer, "Coupled canonical polyadic decompositions and (coupled) decompositions in multilinear rank- $\left(L_{r, n}, L_{r, n}, 1\right)$ terms-part I: Uniqueness," SIAM J. Matrix Anal. Appl., vol. 36, no. 2, pp. 496-522, Apr. 2015.

[24] R. Bro, Multi-way analysis in the food industry: models, algorithms, and applications, Ph.D. thesis, Chemometrics Group, Food Technology. Department of Dairy and Food Science. Royal Veterinary and Agricultural University, Denmark, 1998.

[25] J. W. Brewer, "Kronecker products and matrix calculus in system theory," IEEE Trans. Circuits Syst., vol. CAS-25, no. 9, pp. 772-781, Sep. 1978. 